\title{
Parameters On-line Identification of Dual Three Phase Induction Motor by Voltage Vector Injection in Harmonic Subspace
}

\author{
Shuang Sheng *, Haifeng Lu *, Wenlong Qu *, Ruijie Guo **, and Jinlei Yang***
}

\begin{abstract}
This paper introduces a novel method of on-line identifying the stator resistance and leakage inductance of dual three phase induction motor (DTPIM). According to the machine mathematical model, the stator resistance and leakage inductance can be estimated using the voltage and current values in harmonic subspace. Thus a method of voltage vector injection in harmonic subspace (VVIHS) is proposed, which causes currents in harmonic space. Then the errors between command and actual harmonic currents are utilized to regulate the machine parameters, including stator resistance and leakage inductance. The principle is presented and analyzed in detail. Experimental results prove the feasibility and validity of proposed method.
\end{abstract}

Keywords: On-line identification, Stator resistance, Voltage vector injection, Harmonic subspace, Dual three phase induction motor

\section{Introduction}

The accurate knowledge of stator resistance is important in speed sensor-less vector control [1] and direct torque control (DTC) system [2], which relies on utilization of an induction motor model and requires almost all the motor parameters. The mismatch between the actual value and value used in the speed estimator and DTC system may lead performance deterioration and even instability [3]. Besides, the stator resistance changes significantly with operation temperature. Therefore, an algorithm for on-line identification of the stator resistance is needed.

The available on-line schemes include steady state model calculation method [4], model reference adaptive system (MRAS) [5] and artificial intelligence techniques [6]. All of them designed for the three phase induction motor can mostly be used by the dual three phase system. However, this paper proposes a special method only applicable to the dual three phase induction motor (DTPIM) By injecting voltage vector in harmonic subspace, a clockwise rotated current vector can be detected by transforming the stator currents to harmonic space. Thus the

\footnotetext{
* State Key Lab of Power System, Dept. of Electrical Engineering, Tsinghua University, Beijing, China. (chengs08@mails.tsinghua. edu.cn)

** College of Electrical Engineering, Northeast Dianli University, Jilin Province, China. (ruijieguo666@163.com)

*** Beijing SinoEV Tech Co., Ltd, Daxing District, Beijing, China. (yangjinlei163@163.com)

Received 09 July 2013; Accepted 19 August 2013
}

stator resistance and leakage inductance can be calculated. Experiments are carried out to validate the presented method.

\section{Principle of Voltage Vector Injection Method}

\subsection{Mathematical Model of DPTIM}

The voltage equations of the machine in the $\alpha-\beta-z_{1}-z_{2}-o_{1^{-}}$ $o_{2}$ reference frame can be derived under the following assumptions [7]:

1) The distribution of machine windings is sinusoidal.

2) Flux path is linear.

3) Mutual leakage inductances are neglectable.

The stator and rotor voltage equations in $\alpha-\beta$ subspace are:

$$
\begin{aligned}
{\left[\begin{array}{l}
u_{s \alpha} \\
u_{s \beta} \\
0 \\
0
\end{array}\right]=\left[\begin{array}{cccc}
R_{s} & 0 & 0 & 0 \\
0 & R_{s} & 0 & 0 \\
0 & \omega_{r} L_{m} & R_{r} & \omega_{r} L_{r} \\
-\omega_{r} L_{m} & 0 & -\omega_{r} L_{r} & R_{r}
\end{array}\right]\left[\begin{array}{l}
i_{s \alpha} \\
i_{s \beta} \\
i_{r \alpha} \\
i_{r \beta}
\end{array}\right] } \\
+\left[\begin{array}{cccc}
L_{s} & 0 & L_{m} & 0 \\
0 & L_{s} & 0 & L_{m} \\
L_{m} & 0 & L_{r} & 0 \\
0 & L_{m} & 0 & L_{r}
\end{array}\right]\left[\begin{array}{l}
\frac{i_{s \alpha}}{d t} \\
i_{s \beta} \\
i_{r \alpha} \\
i_{r \beta}
\end{array}\right]
\end{aligned}
$$

In (1), $R_{\mathrm{s}}$ and $R_{\mathrm{r}}$ are stator and rotor resistance, $L_{\mathrm{s}}$ and $L_{\mathrm{r}}$ 
are stator and rotor inductance respectively. $L_{\mathrm{m}}$ is mutual inductance. $\omega_{\mathrm{r}}$ represents the rotor electrical angular frequency.

The stator and rotor equations in $z_{1}-z_{2}$ subspace are:

$$
\begin{aligned}
& \frac{d}{d t}\left[\begin{array}{l}
i_{s z 1} \\
i_{s z 2}
\end{array}\right]=\left[\begin{array}{rr}
-\frac{R_{s}}{L_{l s}} & \\
& -\frac{R_{s}}{L_{l s}}
\end{array}\right]\left[\begin{array}{l}
i_{s z 1} \\
i_{s z 2}
\end{array}\right]+\left[\begin{array}{ll}
\frac{1}{L_{l s}} & \\
\frac{d}{d t}\left[\begin{array}{l}
i_{r z 1} \\
i_{r z 2}
\end{array}\right]=\left[\begin{array}{rr}
-\frac{R_{r}}{L_{l r}} & \\
& -\frac{R_{r}}{L_{l r}}
\end{array}\right]\left[\begin{array}{l}
u_{s z 1} \\
u_{s z 2}
\end{array}\right] \\
i_{r z 1}
\end{array}\right]
\end{aligned}
$$

In (2), $L_{l \mathrm{~s}}$ and $L_{l \mathrm{r}}$ are stator and rotor leakage inductance respectively. The equations in $o_{1}-O_{2}$ subspace are unconsidered, because the currents in this plane are zero due to the isolated neutral points of two windings.

While the transformation from original six-dimensional space to $\alpha-\beta-z_{1}-z_{2}-O_{1}-O_{2}$ space, it should be pointed out that the fundamental components of the machine variables are transformed into the $\alpha-\beta$ subspace, which is coincide with the air gap flux rotation plane. These variables produce a rotating $\mathrm{MMF}$ in the machine air gap and thus the electromagnetic torque is generated. Harmonics with $k=6 m \pm 1,(m=1,3,5, \ldots)$, i.e., the $5^{\text {th }}, 7^{\text {th }}, 17^{\text {th }}, 19^{\text {th }}, \ldots$ harmonics, usually caused by dead time effect and overmodulation strategy application, are mapped into the $z_{1}-z_{2}$ subspace, which is thus called harmonic subspace. As $z_{1}-z_{2}$ space is orthogonal to $\alpha-\beta$ space, the variables on this plane are non-electromechanical energy conversion related.

From (2), the stator resistance and leakage inductance can be calculated if the voltage and current values in harmonic subspace are acquired. The problem is how to generate voltages in $z_{1}-z_{2}$ harmonic subspace.

\subsection{Switching Vectors of Dual Three Phase Machine}

Define the switching signal $S_{x}$ of phase $x(x=a, b, c, u, v$, $w)$.If $S_{x}=1$, it means the inverter upper leg of phase $x$ switches on; Else if $S_{x}=0$, it means the inverter upper leg of phase $x$ switches off. The switching vectors in $\alpha-\beta$ and $z_{1}-z_{2}$ subspace are calculated as below:

$$
\begin{aligned}
\boldsymbol{V}_{\text {switch }}= & {\left[\begin{array}{lllllll}
S_{\alpha} & S_{\beta} & S_{z 1} & S_{z 2} & S_{o 1} & S_{o 2}
\end{array}\right]^{T} } \\
= & U_{d c} \boldsymbol{T}_{6}\left[\begin{array}{llllll}
S_{a} & S_{b} & S_{c} & S_{u} & S_{v} & S_{w}
\end{array}\right]^{T}
\end{aligned}
$$

Where $U_{\mathrm{dc}}$ is the DC voltage, $\boldsymbol{T}_{6}$ is the transforming matrix:

$$
T_{6}=\frac{1}{3}\left[\begin{array}{cccccc}
1 & -\frac{1}{2} & -\frac{1}{2} & \frac{\sqrt{3}}{2} & -\frac{\sqrt{3}}{2} & 0 \\
0 & \frac{\sqrt{3}}{2} & -\frac{\sqrt{3}}{2} & \frac{1}{2} & \frac{1}{2} & -1 \\
1 & -\frac{1}{2} & -\frac{1}{2} & -\frac{\sqrt{3}}{2} & \frac{\sqrt{3}}{2} & 0 \\
0 & -\frac{\sqrt{3}}{2} & \frac{\sqrt{3}}{2} & \frac{1}{2} & \frac{1}{2} & -1 \\
1 & 1 & 1 & 0 & 0 & 0 \\
0 & 0 & 0 & 1 & 1 & 1
\end{array}\right]
$$

Thus the switching vectors can be plot in $\alpha-\beta$ and $z_{1}-z_{2}$ subspace as shown in Fig. 1. Here is a deduced conclusion that the switching vector of DTPIM is composed of switching vector of $\mathrm{ABC}$ windings and switching vector of UVW windings. For instance, the switching vector $V_{100100}$ is composed of $\mathrm{V}_{100 \mathrm{Xxx}}$ of $\mathrm{ABC}$ windings and $\mathrm{V}_{\mathrm{XXX100}}$ of UVW windings. The 'XXX' is ' 000 ' or ' 111 '. That means the reference voltage vector in $\alpha-\beta$ or $z_{1}-z_{2}$ subspace can be decomposed into two reference voltage vectors of two three phase windings, thus a traditional pulse width modulation (PWM) method of three phase induction motors can be applied to simplify the control program.

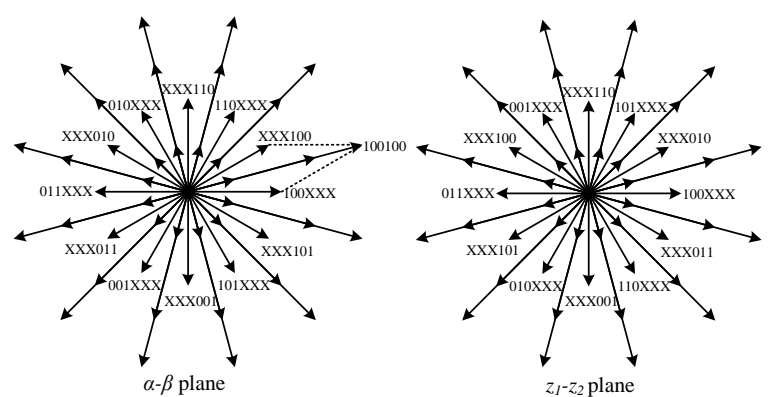

Fig. 1. The distribution of switching vectors in $\alpha-\beta$ and $z 1-$ z2 planes.

\subsection{Voltage Vector Injection in Harmonic Subspace}

Suppose the reference voltage $\boldsymbol{U}_{\text {ref }}=\left(u_{s \alpha}{ }^{*}, u_{s \beta}{ }^{*}\right)$ on $\alpha-\beta$ plane is calculated in the motor control system based on the operation condition, including rotor speed, electromagnetic torque, and flux and so on, it can be decomposed into the vector $\boldsymbol{U}_{a b c}$ and $\boldsymbol{U}_{u v w}$, which are reference voltage vectors of $\mathrm{ABC}$ windings and UVW windings respectively, i.e.:

$$
\boldsymbol{U}_{\text {ref }}=\boldsymbol{U}_{a b c}+\boldsymbol{U}_{u v w}
$$

According to the PWM strategy of three phase machine, the adjacent switching vectors can be chosen as shown in Fig. 2 (a) to satisfy (6). $T_{1}, T_{2}, T_{3}$, and $T_{4}$ are the instants of switching vectors $\boldsymbol{U}_{32}, \boldsymbol{U}_{48}, \boldsymbol{U}_{4}$ and $\boldsymbol{U}_{6} . T_{\mathrm{s}}$ is the sample 
period.

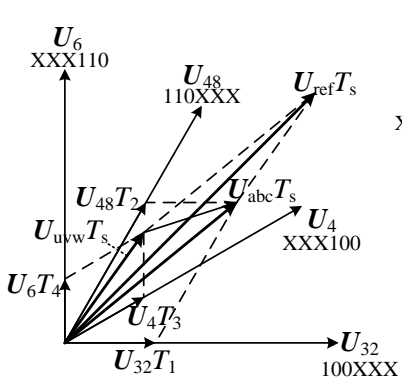

(a) $\alpha-\beta$ plane

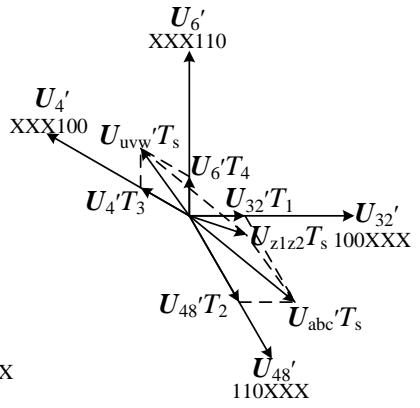

(b) $z_{1}-z_{2}$ plane
Fig. 2. Composed voltage vectors in $\alpha-\beta$ and z1-z2 planes.

$$
\begin{aligned}
& U_{a b c} T_{\mathrm{s}}=\boldsymbol{U}_{32} T_{1}+\boldsymbol{U}_{48} T_{2} \\
& \boldsymbol{U}_{u v w} T_{\mathrm{s}}=U_{4} T_{3}+U_{6} T_{4}
\end{aligned}
$$

Once the switching vectors and instants are decided, the equivalent output voltage vectors of inverter in $\alpha-\beta$ and $z_{1}-z_{2}$ subspace are fixed. Noticed that $\boldsymbol{U}_{32}=\boldsymbol{U}_{32}{ }^{\prime}, \boldsymbol{U}_{6}=\boldsymbol{U}_{6}{ }^{\prime}, \boldsymbol{U}_{48}$ and $\boldsymbol{U}_{48}{ }^{\prime}$ are laterally symmetric, $\boldsymbol{U}_{4}$ and $\boldsymbol{U}_{4}^{\prime}$ are vertically symmetric. The reference voltage vectors of $\mathrm{ABC}$ and UVW windings in $z_{1}-z_{2}$ subspace are composed as the below equation:

$$
\begin{aligned}
& \boldsymbol{U}_{a b c}{ }^{\prime} T_{\mathrm{s}}=\boldsymbol{U}_{32}{ }^{\prime} T_{1}+\boldsymbol{U}_{48}{ }^{\prime} T_{2} \\
& \boldsymbol{U}_{u v w}{ }^{\prime} T_{\mathrm{s}}=\boldsymbol{U}_{4}{ }^{\prime} T_{3}+\boldsymbol{U}_{6}{ }^{\prime} T_{4}
\end{aligned}
$$

Finally the output voltage vector $\boldsymbol{U}_{z 1 z_{2}}$ in $z_{1}-z_{2}$ subspace is composed by $\boldsymbol{U}_{\mathrm{abc}}{ }^{\prime}$ and $\boldsymbol{U}_{\mathrm{uvw}}{ }^{\prime}$.

$$
\boldsymbol{U}_{z 1 z 2}=\boldsymbol{U}_{a b c}{ }^{\prime}+\boldsymbol{U}_{u v w}{ }^{\prime}
$$

Based on the geometrical relationship shown in Fig. 2 (b), $\boldsymbol{U}_{z 1 z 2}$ is vertically symmetric with the error vector of $\boldsymbol{U}_{\mathrm{abc}}$ and $\boldsymbol{U}_{\mathrm{uvw}}$. Define the error vector $\Delta \boldsymbol{U}$ :

$$
\begin{aligned}
\Delta \boldsymbol{U} & =\boldsymbol{U}_{a b c}-\boldsymbol{U}_{u v w}=2 \boldsymbol{U}_{a b c}-\boldsymbol{U}_{\text {ref }} \\
& =\boldsymbol{U}_{\text {ref }}-2 \boldsymbol{U}_{u v w}=\left(\Delta u_{s \alpha}, \Delta u_{s \beta}\right)
\end{aligned}
$$

And if $\boldsymbol{U}_{z 1 z 2}=\left(u_{s z 1}, u_{s z 2}\right)$, yield the below equation:

$$
\begin{aligned}
& \Delta u_{s \alpha}=u_{s z 1} \\
& \Delta u_{s \beta}=-u_{s z 2}
\end{aligned}
$$

The reference voltages of two windings are derived combining (5), (9) and (10) as below:

$$
\begin{aligned}
\boldsymbol{U}_{a b c} & =\boldsymbol{U}_{\mathrm{ref}} / 2+\Delta \boldsymbol{U} / 2=\left(\frac{u_{s \alpha}^{*}+\Delta u_{s \alpha}}{2}, \frac{u_{s \beta}^{*}+\Delta u_{s \beta}}{2}\right) \\
& =\left(\frac{u_{s \alpha}^{*}+u_{s z 1}}{2}, \frac{u_{s \beta}^{*}-u_{s z 2}}{2}\right) \\
\boldsymbol{U}_{u v w} & =\boldsymbol{U}_{\mathrm{ref}} / 2-\Delta \boldsymbol{U} / 2=\left(\frac{u_{s \alpha}^{*}-\Delta u_{s \alpha}}{2}, \frac{u_{s \beta}^{*}-\Delta u_{s \beta}}{2}\right) \\
& =\left(\frac{u_{s \alpha}^{*}-u_{s z 1}}{2}, \frac{u_{s \beta}^{*}+u_{s z 2}}{2}\right)
\end{aligned}
$$

The control diagram of the voltage vector injection in harmonic subspace is shown in Fig. 3. $\boldsymbol{T}\left(\theta_{0}\right)$ is the clockwise rotation transforming matrix. $\theta_{0}$ is the space angle between two windings, which is $30^{\circ}$ in this paper.

$$
\begin{aligned}
{\left[\begin{array}{c}
u_{s \alpha_{-} u v w}^{\prime}{ }^{*} \\
u_{s \beta_{-} u v w}^{\prime}
\end{array}\right] } & =T\left(\theta_{0}\right)\left[\begin{array}{l}
u_{s \alpha_{-} u v w}{ }^{*} \\
u_{s \beta_{-} u v w}^{*}
\end{array}\right] \\
& =\left[\begin{array}{cc}
\cos 30^{\circ} & \sin 30^{\circ} \\
-\sin 30^{\circ} & \cos 30^{\circ}
\end{array}\right]\left[\begin{array}{l}
u_{s \alpha_{-} u v w} \\
u_{s \beta_{-} u v w}
\end{array}\right]
\end{aligned}
$$

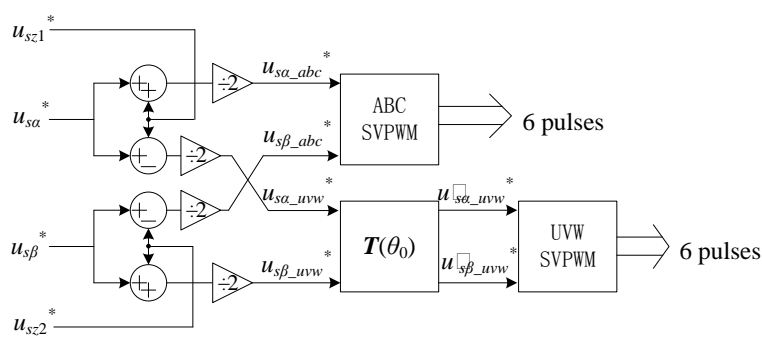

Fig. 3. The control diagram of VVIHS method.

\section{Parameter On-line Identification Method}

\subsection{Stator Resistance and Leakage Inductance On-line Identification Method}

The stator voltage equation in $z_{1}-z_{2}$ frame is transformed to synchronously rotated $m-t$ frame as shown in (13) for simplification. $\omega_{\mathrm{s}}$ is the synchronous electrical angular frequency.

$$
\begin{aligned}
& u_{s m}=R_{s} i_{s m}+\omega_{s} L_{l s} i_{s t}+L_{l s} \frac{d}{d t} i_{s m} \\
& u_{s t}=R_{s} i_{s t}-\omega_{s} L_{l s} i_{s m}+L_{l s} \frac{d}{d t} i_{s t}
\end{aligned}
$$

If $m$-axis is oriented in the direction coincide with the direction of current vector $\boldsymbol{I}_{z 1 z 2}$ in harmonic plane, i.e. $i_{s t}=0$, thus yield the function in steady state: 


$$
\begin{aligned}
& u_{s m}=R_{s} i_{s m} \\
& u_{s t}=-\omega_{s} L_{l s} i_{s m}
\end{aligned}
$$

Suppose the estimated values of stator resistance and stator leakage inductance are $\hat{R}_{s}$ and $\hat{L}_{l s}$ respectively, the reference voltages should be set as shown in (14) to generate a controllable current $\boldsymbol{I}_{z 122}{ }^{*}$ in harmonic plane. Here $\boldsymbol{I}_{z 122}{ }^{*}$ is oriented in $m$-axis, i.e. $\boldsymbol{I}_{z 122}{ }^{*}=i_{s m}{ }^{*}$.

$$
\begin{aligned}
& u_{s m}{ }^{*}=\hat{R}_{s} i_{s m}{ }^{*} \\
& u_{s t}{ }^{*}=-\omega_{s} \hat{L}_{l s} i_{s m}{ }^{*}
\end{aligned}
$$

However, if the estimated values of stator resistance and leakage inductance are inaccurate, the actual current $\boldsymbol{I}_{z 122}$ is different from the reference value $\boldsymbol{I}_{z 1 z^{2}}{ }^{*}$, thus yield:

$$
\begin{aligned}
& u_{s m}{ }^{*}=R_{s} i_{s m}+\omega_{s} L_{l s} i_{s t} \\
& u_{s t}{ }^{*}=R_{s} i_{s t}-\omega_{s} L_{l s} i_{s m}
\end{aligned}
$$

Define the impedance angle $\varphi$ and its estimated value $\hat{\varphi}$ :

$$
\varphi=\arctan \frac{\omega_{s} L_{l s}}{R_{s}}, \quad \hat{\varphi}=\arctan \frac{\omega_{s} \hat{L}_{l s}}{\hat{R}_{s}}
$$

Combine (14), (15) and (16) yield:

$$
\tan \hat{\varphi}-\tan \varphi=-\frac{\omega_{s}^{2} \hat{L}_{l s}^{2}+\hat{R}_{s}^{2}}{R_{s} \hat{R}_{s}\left|\boldsymbol{I}_{z 1 z 2}\right|^{2}}\left|\boldsymbol{I}_{z 1 z 2}^{*}\right| i_{s t}=C i_{s t}
$$

where $C=-\frac{\omega_{s}^{2} \hat{L}_{l s}^{2}+\hat{R}_{s}^{2}}{R_{s} \hat{R}_{s}\left|\boldsymbol{I}_{z 1 z 2}\right|^{2}}\left|\boldsymbol{I}_{z 1 z 2}{ }^{*}\right|<0$, the conclusions are derived from (17) as below:

1) If $i_{s t}<0$, then $\tan \hat{\varphi}>\tan \varphi$, i.e. $\hat{\phi}>\phi$;

2) If $i_{s t}>0$, then $\tan \hat{\varphi}<\tan \varphi$, i.e. $\hat{\phi}<\phi$;

3) If $i_{s l}=0$, only if $\tan \hat{\varphi}=\tan \varphi$, i.e. $\hat{\phi}=\phi$;

The geometrical relationship shown in Fig. 4 gives an intuitive grasp of the idea that the impedance angle could be adjusted by the actual value of harmonic space current in $t$-axis. A PI regulator is utilized to adjust the tangent value of impedance angle in (18).

$$
\tan \hat{\varphi}=\tan \varphi_{0}+k_{p 1} i_{s t}+k_{i 1} \int i_{s t} d t
$$

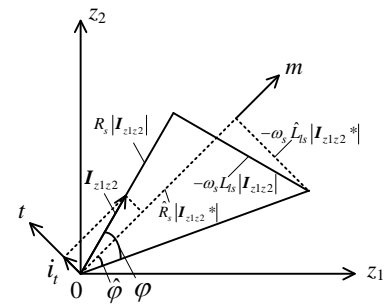

(a) $\hat{\varphi}>\varphi$

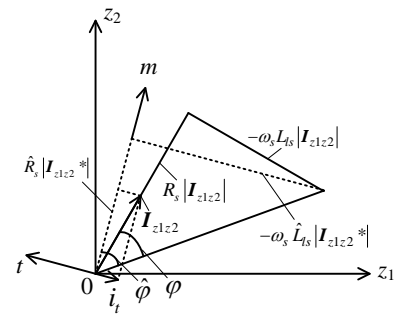

(b) $\hat{\varphi}<\varphi$
Fig. 4. The currents and voltages in $\mathrm{z} 1-\mathrm{z} 2$ plane.

$\varphi_{0}$ is the initial value of estimated impedance angle. When $\hat{\varphi}=\varphi, i_{s l}=0, R_{s}=u_{s m}{ }^{*} / i_{s m}, \hat{R}_{s}=u_{s m}{ }^{*} / i_{s m}{ }^{*}$, the obtained error of estimated stator resistance is:

$$
\begin{aligned}
\Delta \hat{R}_{s} & =\hat{R}_{s}-R_{s}=\frac{u_{s m}{ }^{*}}{i_{s m}{ }^{*}}-\frac{u_{s m}{ }^{*}}{i_{s m}}=\frac{i_{s m}-i_{s m}{ }^{*}}{i_{s m}{ }^{*} i_{s m}} u_{s m}{ }^{*} \\
& =-\frac{\Delta i_{s m}}{i_{s m}{ }^{*} i_{s m}} u_{s m}{ }^{*}
\end{aligned}
$$

(19) explains that:

1) If $i_{s m}<i_{s m}{ }^{*}$, then $\hat{R}_{s}<R_{s}$;

2) If $i_{s m}>i_{s m}{ }^{*}$, then $\hat{R}_{s}>R_{s}$;

3) If $i_{s m}=i_{s m}{ }^{*}$, only if $\hat{R}_{s}=R_{s}$;

Similarly a PI regulator is utilized to adjust the stator resistance value by the error of actual and reference value of harmonic current in $m$-axis. The equation is shown as below:

$$
\hat{R}_{s}=R_{s 0}+k_{p 2}\left(i_{s m}{ }^{*}-i_{s m}\right)+k_{i 2} \int\left(i_{s m}{ }^{*}-i_{s m}\right) d t
$$

The diagram block of parameter on-line identification is shown in Fig. 5. The $i_{s m}{ }^{*}$ is the command value, to control the magnitude of the current in harmonic plane. The $i_{s m}$ and $i_{s t}$ is transformed from the six phase currents by matrix $\boldsymbol{T}_{6}$. The $u_{s z 1}{ }^{*}$ and $u_{s z 2}{ }^{*}$ are calculated in (14) utilizing the estimated value of stator resistance and leakage inductance. Then they are used to calculate the reference voltages for $\mathrm{ABC}$ and UVW windings as shown in Fig. 3.

\subsection{Dead Time Compensation Strategy}

The distorted voltage caused by dead-time effect can generate $5^{\text {th }}$ and $7^{\text {th }}$ harmonics in phase currents, which will be transformed into $z_{1}-z_{2}$ subspace. That degrades the dynamic and static performance of parameters adjusting regulator. Thus the dead-time should be compensated. 


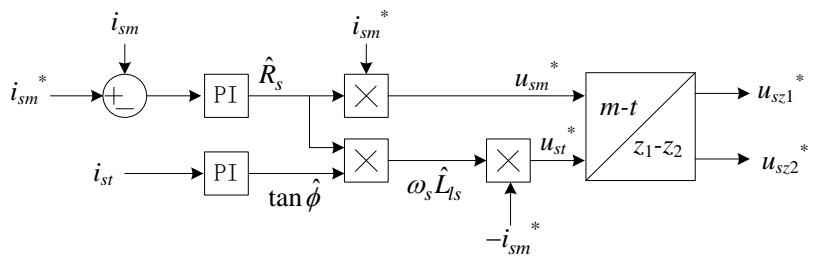

Fig. 5. The diagram block of parameter on-line identification.

As known that the estimation of current polarity is very important for dead-time compensation, especially around the zero-crossing point, thus the currents $i_{s \alpha}$ and $i_{s \beta}$ are utilized to reconstruct the fundamental wave of six phase currents.

$$
\left[\begin{array}{llllll}
i_{a 1} & i_{b 1} & i_{c 1} & i_{u 1} & i_{v 1} & i_{w 1}
\end{array}\right]^{\mathrm{T}}=\boldsymbol{T}_{6}^{-1}\left[\begin{array}{llllll}
i_{s \alpha} & i_{s \beta} & 0 & 0 & 0 & 0
\end{array}\right]^{\mathrm{T}}
$$

Because $\alpha-\beta$ subspace mainly contains the fundamental part of the signals, therefore the calculated currents in (21) is approximately sinusoid. Then the current polarity could be judged as below:

$$
\operatorname{sgn}\left[i_{x 1}\right]=\left\{\begin{array}{cc}
1, & i_{x 1}>0 \\
-1, & i_{x 1}<0
\end{array} \quad(x=a, b, c, u, v, w)\right.
$$

\subsection{Current Command in Harmonic Subspace}

There is a problem of the dead-time compensation method mentioned before: the waveform of reconstructed fundamental phase currents will be distorted if the relative angles of six phase currents change due to generated harmonic current $\boldsymbol{I}_{z 1 z 2}$. That will deteriorate the dead-time compensation performance. Thus the current $\boldsymbol{I}_{z 1 z 2}$ in $z_{1}-z_{2}$ subspace should be controlled to maintain the phase position of phase currents.

Suppose the output currents of DTPIM after applying the proposed voltage vector injection strategy are:

$$
\begin{aligned}
& i_{s a}=I_{1} \cos \theta \\
& i_{s b}=I_{1} \cos (\theta-2 \pi / 3) \\
& i_{s c}=I_{1} \cos (\theta-4 \pi / 3) \\
& i_{s u}=I_{2} \cos (\theta-\pi / 6) \\
& i_{s v}=I_{2} \cos (\theta-5 \pi / 6) \\
& i_{s w}=I_{2} \cos (\theta-3 \pi / 2)
\end{aligned}
$$

(23) shows that the magnitudes of two windings are different but the relative angles are unchanged. Transform (23) to $\alpha-\beta-z_{1}-z_{2}$ frame yield:

$$
\begin{aligned}
& i_{s \alpha}=\frac{I_{1}+I_{2}}{2} \cos \theta \\
& i_{s \beta}=-\frac{I_{1}+I_{2}}{2} \sin \theta \\
& i_{s z 1}=\frac{I_{1}-I_{2}}{2} \cos \theta \\
& i_{s z 2}=\frac{I_{1}-I_{2}}{2} \sin \theta
\end{aligned}
$$

Or:

$$
\begin{aligned}
& i_{s z 1}=\frac{I_{1}-I_{2}}{2} \frac{i_{s \alpha}}{i_{s \alpha}{ }^{2}+i_{s \beta}{ }^{2}} \\
& i_{s z 2}=-\frac{I_{1}-I_{2}}{2} \frac{i_{s \beta}}{i_{s \alpha}{ }^{2}+i_{s \beta}{ }^{2}}
\end{aligned}
$$

Therefore the current commands in harmonic plane could be set as (26) to maintain the phase position of stator currents.

$$
\begin{aligned}
& i_{s z 1}{ }^{*}=\left|\boldsymbol{I}_{z 1 z 2}{ }^{*}\right| \frac{i_{s \alpha}}{i_{s \alpha}{ }^{2}+i_{s \beta}{ }^{2}} \\
& i_{s z 2}{ }^{*}=-\left|\boldsymbol{I}_{z 1 z 2}{ }^{*}\right| \frac{i_{s \beta}}{i_{s \alpha}{ }^{2}+i_{s \beta}{ }^{2}}
\end{aligned}
$$

If the $m$-axis is oriented in the direction of $\boldsymbol{I}_{z 1 z 2}{ }^{*}$, then the transforming equation from $z_{1}-z_{2}$ frame to $m-t$ frame is defined as below:

$$
\begin{aligned}
{\left[\begin{array}{c}
x_{m} \\
x_{t}
\end{array}\right]=T(\gamma)\left[\begin{array}{c}
x_{z 1} \\
x_{z 2}
\end{array}\right] } \\
=\left[\begin{array}{cc}
\cos \gamma & \sin \gamma \\
-\sin \gamma & \cos \gamma
\end{array}\right]\left[\begin{array}{l}
x_{z 1} \\
x_{z 2}
\end{array}\right]
\end{aligned}
$$

Where $\gamma$ is the angle of $\boldsymbol{I}_{z 1 z 2}{ }^{*}, \gamma=\arctan \left(i_{s z 2}{ }^{*} / i_{s z 1}{ }^{*}\right)$.

\section{Experimental Results}

To evaluate the feasibility and validity of proposed method, the tests are performed on a dual three phase induction motor with rated power $2.5 \mathrm{KW}$ and rated speed 1000r.min ${ }^{-1}$. The TMSF2812 DSP is used as main control processor and the switching frequency is $5 \mathrm{KHz}$, DC link voltage is $100 \mathrm{~V}$.

The diagram block of DTPIM control system utilizing the proposed VVIHS method to identify the stator resistance and leakage inductance is shown in Fig. 6. 


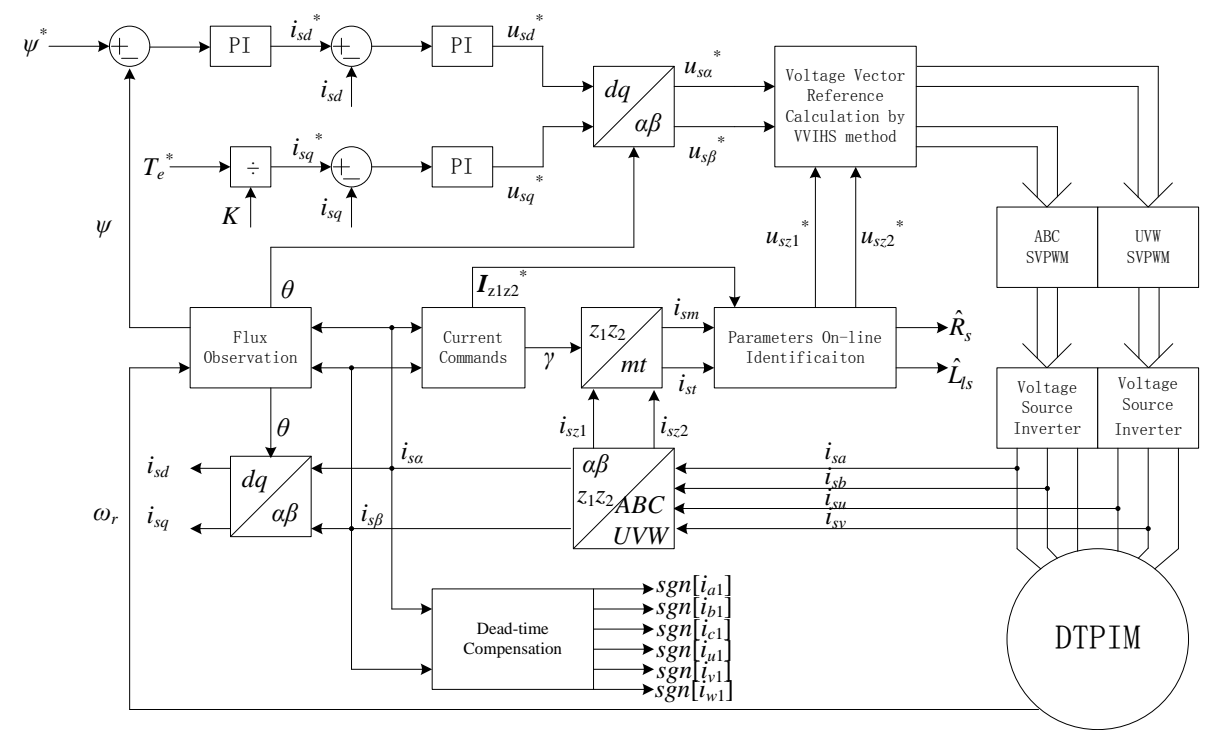

Fig. 6. The diagram block of DTPIM control system utilizing VVIHS method to on-line identify machine parameters.
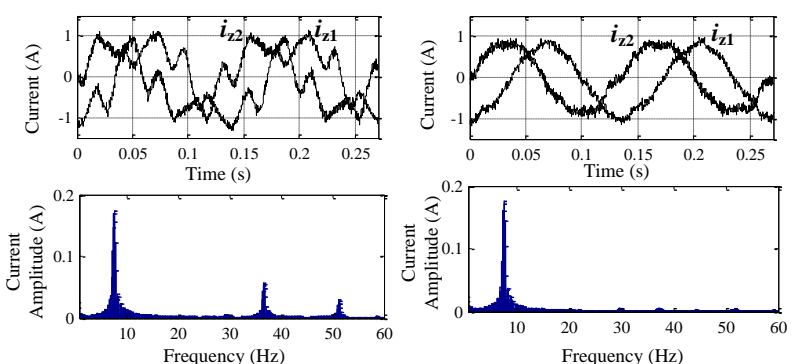

(a) without dead-time compensation (b) with dead-time compensation

Fig. 7. The waveforms and harmonic analysis of currents in z1-z2 plane.

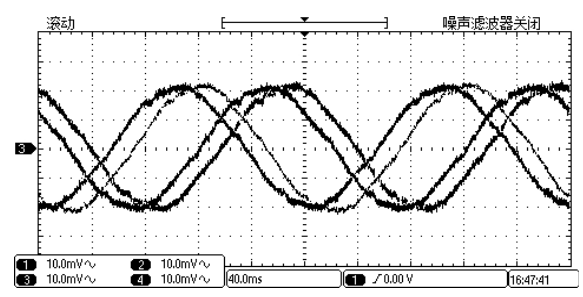

(a) without VVIHS

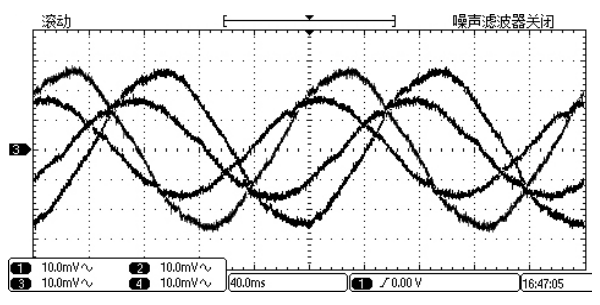

(b) with VVIHS

Fig. 8. The waveforms of phase currents of DTPIM.

Fig. 7 shows the current waveforms and harmonic analysis in $z_{1}-z_{2}$ subspace. It is clear that the $5^{\text {th }}$ and $7^{\text {th }}$

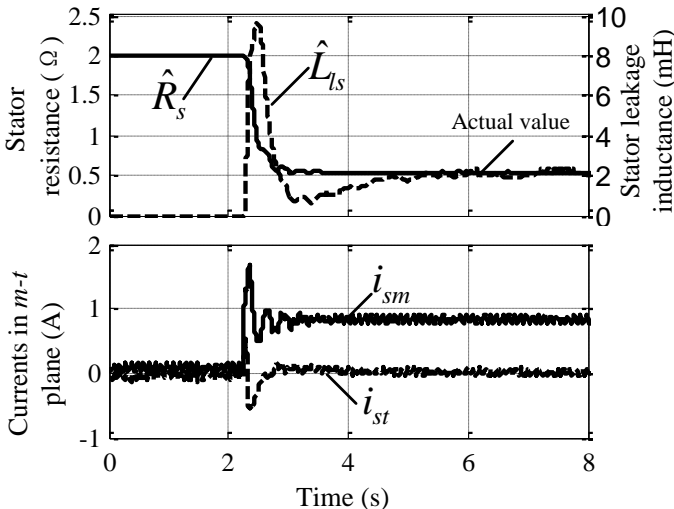

(a) $\hat{R}_{s 0}>R_{s}, \quad \hat{L}_{l s 0}<L_{l s}$
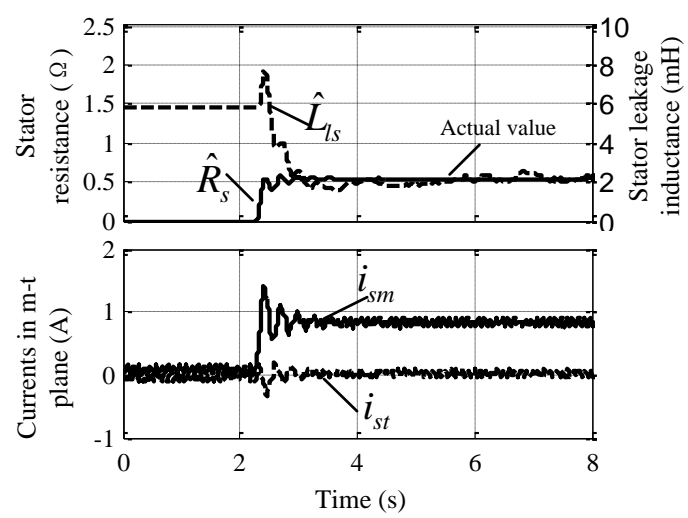

(b) $\hat{R}_{s 0}<R_{s}, \quad \hat{L}_{l s 0}>L_{l s}$

Fig. 9. The experimental results of parameter on-line identification method.

harmonics are eliminated since the dead-time compensation strategy has been applied, which can improve the accuracy and stability of identification method. 
Fig. 8 shows the current waveforms of phase A, B, U, V. It proves that the relative angles of stator currents keep unchanged with or without applying VVIHS method. Thus the dead-time is exactly compensated, which improves the quality of phase currents.

Fig. 9 shows the experimental results of parameters online identification method. The accurate values of stator resistance and leakage inductance are rapidly acquired in less than one second. The currents $i_{s m}$ and $i_{s t}$ also converge to command values fast and accurate. The experimental results prove the feasibility and validity of proposed method.

\section{Conclusion}

In this paper, a novel method of parameters on-line identification of dual three phase induction motor is proposed. By injecting voltage vector in harmonic subspace, the stator resistance and leakage inductance can be estimated fast and accurately. This method relies on none of the other parameters of machine such as rotor resistance and mutual inductance, and the dead-time is compensated to improve the identification accuracy. Besides, it is easy to be programmed and installed in control system to improve the control performance.

\section{References}

[1] Rajashekara K, Kawamura A, Matsuse K, "Sensorless Control of AC Motor Drives", Piscataway, NJ: IEEE Press, 1996.

[2] Takahashi I, Noguchi T, "A new quick response and high efficiency control strategy of an induction motor," IEEE Transaction on Industry Applications, vol. 22, no. 5, pp. 820 827, 1986.

[3] Armstrong G J, Atkinson D J, "A comparison of model reference adaptive system and extended Kalman filter estimation for sensorless vector drives," European Conference on Power Electronics and Applications, Trondheim, Norway, 1997.

[4] Ha I J, Lee S H, “An online identification method for both stator and rotor resistance of induction motors without rotational transducers," IEEE Transactions on Industrial Electronics, vol. 47, pp. 842-853, 2000.

[5] Zhen L, Xu L, "Sensorless field orientation control of induction machines based on mutual MRAS scheme," IEEE Transactions on Industrial Electronics, vol. 45, pp. 824-831, 1998.

[6] Cabrera L A, Elbuluk E, Hsain I, "Tuning the stator resistance of induction motors using artificial neural network," IEEE Transactions on Power Electronics, vol. 12 pp. 779-787, 1997.

[7] Zhao Y F, Lipo T A, "Space vector PWM control of dual three-phase induction machine using vector space decomposition," IEEE Transaction on Industry Applications, vol. 31, no. 5, pp. 1100-1109, 1995.

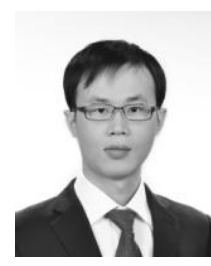

Shuang Sheng received the B.S degree in electrical engineering from Tsinghua University in 2008. Since then he has been with the department of electrical engineering in Tsinghua University as a Ph.D. student. His current major research interests are power electronics and induction motor drives.

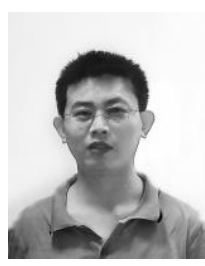

Haifeng Lu received the B.S degree and M.S. degree from Southeast University in 1998 and 2001 respectively, and the Ph.D. degree from the Tsinghua University in 2005, all in electrical engineering. Since 2005, he has been with the department of electrical engineering in Tsinghua University, serving as an assistant professor. His current major research interests are in power electronics and motor drives.

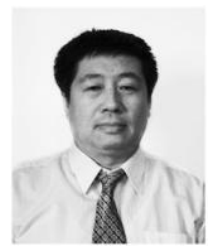

Wenglong Qu received the B.S. degree from the Department of Electrical Engineering, Tsinghua University, in 1970. Since 1970, he has been a teacher in the Department of Electrical Engineering, Tsinghua University, where he is currently a professor. $\mathrm{He}$ is the author of more than 80 technical papers in the fields of power electronics and motor control. His research interests include ac and dc motor controls, dcdc converters, soft-switching techniques, electric vehicle drives and power steering system control.

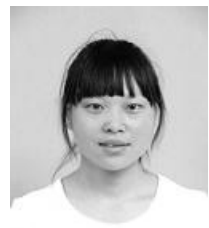

Ruijie Guo received the B.S degree in electrical engineering from Northeast Dianli University in 2011. Since then she has been with the department of electrical engineering in Northeast Dianli University as a Master student. Her current major research interests are power electronics and induction motor drives.

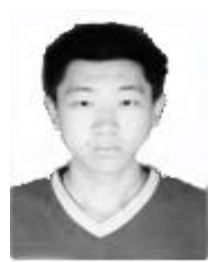

Jinlei Yang received the B.S degree and M.S. degree from Harbin University of Science and Technology in 2006 and 2009, respectively, all in electrical engineering. He works at SinoEV Tech Co, Ltd engaged in electric vehicle drives, since 2011. His research interests are induction motor Vector controls. 